\title{
Gemcitabine, Oxaliplatin and Bevacizumab in patients with biliary tract cancers
}

\author{
Amit Mahipal ${ }^{1}$, Edith Mitchell ${ }^{2}$ \\ 1. Clinical Research Unit, H. Lee Moffitt Cancer Center and Research Institute, Tampa, FL, USA. 2. Department of Medical \\ Oncology, Thomas Jefferson University, Philadelphia, PA, USA
}

Correspondence: Amit Mahipal. Address: Clinical Research Unit, H. Lee Moffitt Cancer Center, 12902 Magnolia Drive, MCC\#3177, Tampa, FL 33647, USA. E-mail: amit.mahipal@moffitt.org

Received: March 5, 2013

Accepted: April 2, 2013

Online Published: April 9, 2013

DOI : $10.5430 /$ jst.v3n3p29

URL: http://dx.doi.org/10.5430/jst.v3n3p29

\begin{abstract}
Introduction: Biliary tract cancers (BTCs) are relatively rare cancers with poor prognosis. There are few effective chemotherapy regimens available for the treatment of metastatic disease and gemcitabine-based combination therapy is frequently utilized. Vascular endothelial growth factor is expressed in the BTCs and is associated with poor prognosis. Bevacizumab, an antiangiogenic antibody, may play a role in the treatment of metastatic BTCs.

Presentation: We report the outcomes in six patients with BTCs treated with the combination of gemcitabine, oxaliplatin and bevacizumab (GEMOX-B) at Thomas Jefferson University. Three patients had previously been treated with different chemotherapy regimens. Two patients achieved partial response and four patients had stable disease. One patient was able to undergo curative resection. Progression free survival ranged from 6 to 42 months.
\end{abstract}

Conclusions: GEMOX-B is an active regimen in the treatment of metastatic BTCs and needs to be investigated in a randomized clinical trial.

\section{Key words}

Biliary tract neoplasm, Gemcitabine, Oxaliplatin, Bevacizumab

\section{I ntroduction}

Biliary tract cancers (BTCs) comprise of neoplasms arising from the epithelium of the gallbladder and intrahepatic and extrahepatic bile ducts. BTCs are relatively rare cancers with estimated 9,810 new cases diagnosed annually in United States ${ }^{[1]}$. The etiology of BTCs remains unclear with most of the cases occurring in patients with the absence of any known risk factors. Chronic inflammation of biliary ducts, primary sclerosing cholangitis, intrahepatic biliary stones, parasitic infection, and cirrhosis are associated with cholangiocarcinoma ${ }^{[2]}$.

The prognosis of BTCs remains poor with 5-year relative survival of only $15 \%{ }^{[1]}$. Although surgical resection is the only curative option, there is a high rate of recurrences. Post-resection, 5 year relative survival ranges from $8 \%$ to $47 \%$ for intrahepatic cholangiocarcinoma and $20 \%$ to $54 \%$ for distal cholangiocarcinoma ${ }^{[2,3]}$. The relapse rate depends on the location of the tumor, extent of the surgery and negative resection margins. Patients with unresectable or metastatic BTCs have dismal prognosis with the median survival of less than 1 year ${ }^{[4]}$. 
Gemcitabine-based combination chemotherapy is frequently employed in the treatment of BTCs. Several drugs have been tested in combination with gemcitabine to increase its efficacy. The pooled analysis of 104 trials demonstrated that the gemcitabine and platinum combination resulted in higher response rate and tumor control rates and trend towards improved survival ${ }^{[5]}$. Advanced Biliary Cancer- 02 trial, a randomized phase 3 study demonstrated the superiority of the combination of gemcitabine and cisplatin compared to gemcitabine alone in 410 patients ${ }^{[6]}$. The median overall survival was 11.7 months in the combination group as compared to 8.1 months in patients receiving single agent gemcitabine. Several phase II studies have demonstrated that the combination of gemcitabine and oxaliplatin has similar efficacy with favorable toxicity profile ${ }^{[7-9]}$.

Vascular endothelial growth factor (VEGF) is considered a primary angiogenic stimulator and is expressed in BTCs. VEGF expression has been demonstrated to correlate with advanced stage disease and poor prognosis in patients with extrahepatic BTCs and gallbladder cancer ${ }^{[10-12]}$. Bevacizumab is a humanized monoclonal antibody against VEGF and has demonstrated to be active in several tumor types including colorectal cancer, glioblastoma multiforme, and lung cancers. In addition to the direct antiangiogenic effects, bevacizumab may also improve the delivery of chemotherapy by altering tumor vasculature and decreasing the elevated interstitial pressure in tumors ${ }^{[13]}$. A phase II trial testing the addition of bevacizumab to gemcitabine and oxaliplatin (GEMOX-B) was recently reported ${ }^{[14]}$. The overall response rate was $40 \%$ with additional $29 \%$ of patients achieving stable disease. The median progression-free survival was 7 months.

In this retrospective study, we report the outcomes of the patient with BTCs who received GEMOX-B at our institution. This study was approved by the Institutional Review Board at Thomas Jefferson University. All patients were administered Gemcitabine $1000 \mathrm{mg} / \mathrm{m}^{2}$, oxaliplatin $85 \mathrm{mg} / \mathrm{m}^{2}$ and bevacizumab $10 \mathrm{mg} / \mathrm{kg}$ on day 1 and 15 of every 28-day cycle. Table 1 describes the baseline characteristics and clinical outcomes of the patients.

Table 1. Baseline characteristics and clinical outcomes of the patients

\begin{tabular}{|c|c|c|c|c|c|c|c|c|c|c|}
\hline Patient & Age & Race & Sex & Diagnosis & $\begin{array}{l}\text { Prior } \\
\text { Therapy }\end{array}$ & $\begin{array}{l}\text { Number of } \\
\text { Cycles }\end{array}$ & $\begin{array}{l}\text { Best } \\
\text { Response }\end{array}$ & Alive & Progression-Free & $\begin{array}{l}\text { Progression- } \\
\text { Free } \\
\text { Survival } \\
\text { (months) }\end{array}$ \\
\hline 1 & 50 & Asian & $\mathrm{F}$ & $\mathrm{C}$ & No & 9 & PR & Yes & Yes & 42.2 \\
\hline 2 & 45 & White & $\mathrm{F}$ & GB & Yes & 7 & SD & Yes & No & 6.4 \\
\hline 3 & 51 & White & $\mathrm{F}$ & $\mathrm{C}$ & Yes & 9 & SD & No & No & 10.1 \\
\hline 4 & 40 & White & $\mathrm{F}$ & $\mathrm{C}$ & No & 13 & SD & Yes & Yes & 10.2 \\
\hline 5 & 64 & White & M & $\mathrm{C}$ & No & 21 & PR & Yes & Yes & 41.2 \\
\hline 6 & 44 & White & $\mathrm{F}$ & $\mathrm{C}$ & Yes & 7 & SD & Yes & No & 11.7 \\
\hline
\end{tabular}

C: Cholangiocarcinoma; F: Female; GB: Gall Bladder; M: Male; PR: Partial Response; SD: Stable Disease

\section{Case presentations}

\subsection{Patient 1}

A 50-year-old Asian female with a history of hepatitis B presented with a $15.5 \mathrm{~cm} \times 12.6 \mathrm{~cm}$ right hepatic lobe mass. Based on elevated alpha fetoprotein of $1071 \mathrm{mg} / \mathrm{mL}$ and imaging findings, a preliminary diagnosis of hepatocellular carcinoma was made. She began chemotherapy with GEMOX-B and had partial response after 4 cycles. Her tumor size decreased to $3.6 \times 1.7 \mathrm{~cm}$ (Figure 1). She subsequently underwent chemoembolization followed by liver transplant. Pathology revealed poorly differentiated adenocarcinoma consistent with cholangiocarcinoma. She underwent postoperative adjuvant chemotherapy with GEMOX-B for 5 more cycles. She currently has no evidence of disease and remains in complete remission. 
Figure 1. CT scans of the patient 1 demonstrating the response to therapy after 4 cycles

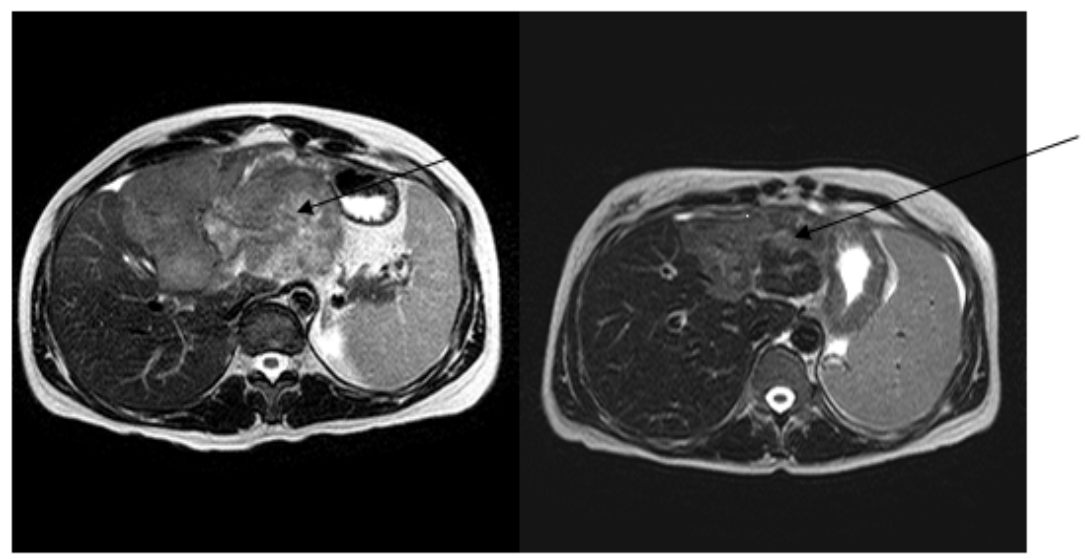

\subsection{Patient 2}

A 45 year old female initially presented with right upper quadrant pain. CT scan demonstrated a dominant mass measuring $7.7 \times 7.1 \mathrm{~cm}$ in right lobe of the liver and two small left upper lobe lung nodules. Liver biopsy was consistent with adenocarcinoma, arising from gallbladder. She initially was treated with carboplatin and paclitaxel for 4 cycles. She had progressive disease and was then referred to our institution. She received GEMOX-B for 7 cycles. She initially had a partial response with decrease in the size of the liver lesion. She progressed after 6.4 months on treatment with GEMOX-B.

\subsection{Patient 3}

A 51 year old white female presented with confluent hepatic mass measuring $16.5 \times 9.2 \mathrm{~cm}$ along with multiple subcentimeter bilateral pulmonary nodules. Ultrasound guided liver biopsy was consistent with cholangiocarcinoma. She was initially treated on a clinical trial with gemcitabine, irinotecan and panitumumab for 3 cycles. She had progressive disease and subsequently received combination chemotherapy with 5-Fluoraouracil, irinotecan, oxaliplatin and bevacizumab. She had disease progression after 4 cycles. She was then treated with GEMOX-B. She had a partial response to therapy. She received 8 cycles. However, she developed autoimmune hemolytic anemia requiring splenectomy. Post-operatively, she received 1 more cycle of chemotherapy. Unfortunately, she developed multifocal pneumonia and expired.

\subsection{Patient 4}

A 40 year old white female was found to have a $13.6 \times 11.7 \mathrm{~cm}$ mass in the left hepatic lobe. Liver biopsy was consistent with cholangiocarcinoma. PET scan demonstrated another hypermetabolic lesion in the lumbar spine. Biopsy of the bone lesion was consistent with adenocarcinoma as well. Colonoscopy was negative. She was treated with GEMOX-B and had decrease in the size of the tumor with measurement of $10.9 \times 10 \mathrm{~cm}$. By RECIST criteria, she had stable disease. She remains on treatment and has completed 13 cycles.

\subsection{Patient 5}

A 64 year old Caucasian male presented with $13.7 \times 9 \mathrm{~cm}$ heterogeneous liver mass and periportal lymphadenopathy. Pathology was consistent with cholangiocarcinoma. Patient received 7 cycles of GEMOX-B. He had a good response to therapy and underwent laparotomy for possible resection. However, tumor implants were found on the diaphragm and resection was not performed. The patient remained off treatment for 6.5 months per his wishes. He had interval increase in the size of the tumor. He was restarted on chemotherapy with GEMOX-B and had partial response. He received 7 cycles. He then again went off treatment for 8 months and subsequently had progressive disease. He was restarted on GEMOX-B regimen with partial response. He has received 7 more cycles and continues on treatment. 


\subsection{Patient 6}

A 45 year old Caucasian female initially presented with right upper quadrant pain. MRI of the abdomen demonstrated multiple hepatic lesions in segment 5 and 6 with largest mass being $7.2 \mathrm{~cm} \times 6.1 \mathrm{~cm}$. Biopsy of the mass was consistent with cholangiocarcinoma with liver metastases. She underwent resection of the tumor, cholecystectomy and resection of segments 5 and 6 of the liver. Post-operatively, she received 5-Fluorouracil based therapy. She developed recurrent disease and was treated with GEMOX-B. She initially had stable disease and completed 7 cycles. She developed progressive disease after 11.7 months and was taken off treatment.

\section{Discussion}

In our series of six patients with BTCs treated with GEMOX-B, two patients achieved partial responses and four patients had stable disease. One patient was able to undergo regression of the tumor to an extent that the tumor became resectable and the patient remains free of malignancy. Three patients had received prior therapies. One patient had gallbladder cancer and others had cholangiocarcinoma. The progression-free survival ranged from 6 to 42 months. The outcome of these patients argues well with the historical data. In the phase II trial reported by Zhu et al, GEMOX-B was tested in 35 patients with BTCs resulting in PFS at 6 months of $63 \%$ and clinical benefit rate (PR+SD) of $69 \%{ }^{[14]}$. However, the study did not meet the pre-defined endpoint of improving 6-month PFS from 50\% to 70\%. The regimen was well tolerated with few grade 3 or 4 adverse events including fatigue, neutropenic fever, anorexia and elevation of liver enzymes.

The prognosis of unresectable or metastatic BTCs remains dismal with poor sensitivity to chemotherapeutic agents. The combination chemotherapy of gemcitabine and a platinum agent is considered the standard therapy with the median survival of less than 12 months. To further improve the efficacy of this regimen, molecular targeted agents have been investigated with limited success ${ }^{[15,16]}$. A phase III trial of gemcitabine and oxaliplatin with or without erlotinib did not demonstrate any difference in PFS or OS ${ }^{[15]}$. The role of cetuximab in addition to gemcitabine and oxaliplatin was tested in a phase II trial of 30 patients ${ }^{[16]}$. The overall response rate was $63 \%$ and median PFS and OS were 8.8 months and 15.2 months respectively. Ninety percent of the patients had tumors that were wild type of KRAS. Despite the fact that the response rates were high, the median PFS and OS was similar to those reported in ABC-02 trial that investigated the combination of gemcitabine and cisplatin ${ }^{[6]}$.

Our report suggests that GEMOX-B is an active regimen in BTCs and warrants further investigation in a larger clinical trial.

\section{Conflict of interests}

The authors declare that they have no conflict of interests.

\section{References}

[1] Siegel R, Naishadham D, Jemal A: Cancer statistics, 2012. CA Cancer J Clin. 2012; 62: 10-29. PMid:22237781 http://dx.doi.org/10.3322/caac.20138

[2] Khan SA, Thomas HC, Davidson BR, Taylor-Robinson SD: Cholangiocarcinoma. Lancet. 2005; 366: 1303-1314. http://dx.doi.org/10.1016/S0140-6736(05)67530-7

[3] de Groen PC, Gores GJ, LaRusso NF, Gunderson LL, Nagorney DM: Biliary tract cancers. N Engl J Med. 1999; 341 : $1368-1378$. PMid:10536130 http://dx.doi.org/10.1056/NEJM199910283411807

[4] Hezel AF, Zhu AX: Systemic therapy for biliary tract cancers. Oncologist. 2008; 13: 415-423. PMid:18448556 http://dx.doi.org/10.1634/theoncologist.2007-0252

[5] Eckel F, Schmid RM: Chemotherapy in advanced biliary tract carcinoma: a pooled analysis of clinical trials. Br J Cancer. 2007; 96: 896-902. PMid:17325704 http://dx.doi.org/10.1038/sj.bjc.6603648 
[6] Valle J, Wasan H, Palmer DH, Cunningham D, Anthoney A, Maraveyas A et al: Cisplatin plus gemcitabine versus gemcitabine for biliary tract cancer. N Engl J Med. 2010; 362: 1273-1281. PMid:20375404 http://dx.doi.org/10.1056/NEJMoa0908721

[7] Andre T, Tournigand C, Rosmorduc O, Provent S, Maindrault-Goebel F, Avenin D et al: Gemcitabine combined with oxaliplatin (GEMOX) in advanced biliary tract adenocarcinoma: a GERCOR study. Ann Oncol. 2004; 15: 1339-1343. PMid:15319238 http://dx.doi.org/10.1093/annonc/mdh351

[8] Harder J, Riecken B, Kummer O, Lohrmann C, Otto F, Usadel H et al: Outpatient chemotherapy with gemcitabine and oxaliplatin in patients with biliary tract cancer. Br J Cancer. 2006; 95: 848-852. PMid:16969352 http://dx.doi.org/10.1038/sj.bjc.6603334

[9] Jang JS, Lim HY, Hwang IG, Song HS, Yoo N, Yoon S et al: Gemcitabine and oxaliplatin in patients with unresectable biliary cancer including gall bladder cancer: a Korean Cancer Study Group phase II trial. Cancer Chemother Pharmacol. 2010; 65: 641-647. PMid:19652971 http://dx.doi.org/10.1007/s00280-009-1069-7

[10] Nakashima T, Kondoh S, Kitoh H, Ozawa H, Okita S, Harada T et al: Vascular endothelial growth factor-C expression in human gallbladder cancer and its relationship to lymph node metastasis. Int J Mol Med. 2003; 11: 33-39. PMid:12469214

[11] Hida Y, Morita T, Fujita M, Miyasaka Y, Horita S, Fujioka Y et al: Vascular endothelial growth factor expression is an independent negative predictor in extrahepatic biliary tract carcinomas. Anticancer Res. 1999; 19: 2257-2260. PMid:10472340

[12] Giatromanolaki A, Koukourakis MI, Simopoulos C, Polychronidis A, Sivridis E: Vascular endothelial growth factor (VEGF) expression in operable gallbladder carcinomas. Eur J Surg Oncol. 2003; 29: 879-883. PMid:14624781 http://dx.doi.org/10.1016/j.ejso.2003.09.013

[13] Jain RK: Normalizing tumor vasculature with anti-angiogenic therapy: a new paradigm for combination therapy. Nat Med. 2001; 7: 987-989. PMid:11533692 http://dx.doi.org/10.1038/nm0901-987

[14] Zhu AX, Meyerhardt JA, Blaszkowsky LS, Kambadakone AR, Muzikansky A, Zheng H et al: Efficacy and safety of gemcitabine, oxaliplatin, and bevacizumab in advanced biliary-tract cancers and correlation of changes in 18-fluorodeoxyglucose PET with clinical outcome: a phase 2 study. Lancet Oncol. 2010; 11: 48-54. http://dx.doi.org/10.1016/S1470-2045(09)70333-X

[15] Lee J, Park SH, Chang HM, Kim JS, Choi HJ, Lee MA et al: Gemcitabine and oxaliplatin with or without erlotinib in advanced biliary-tract cancer: a multicentre, open-label, randomised, phase 3 study. Lancet Oncol. 2012; 13: 181-188.

http://dx.doi.org/10.1016/S1470-2045(11)70301-1

[16] Gruenberger B, Schueller J, Heubrandtner U, Wrba F, Tamandl D, Kaczirek K et al: Cetuximab, gemcitabine, and oxaliplatin in patients with unresectable advanced or metastatic biliary tract cancer: a phase 2 study. Lancet Oncol. 2010; 11: 1142-1148. http://dx.doi.org/10.1016/S1470-2045(10)70247-3 